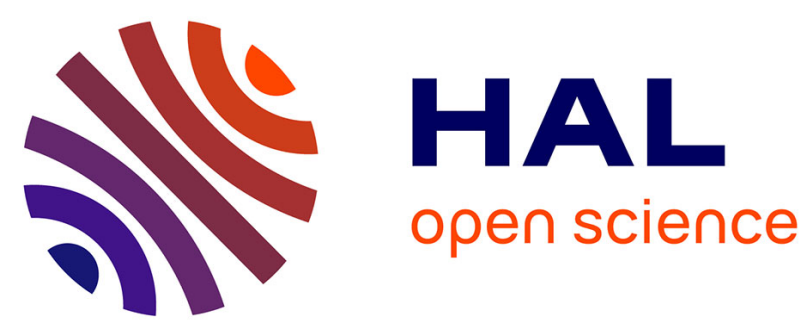

\title{
Dynamic 3D Scene Reconstruction and Enhancement
} Cansen Jiang, Yohan Fougerolle, David Fofi, Cédric Demonceaux

\section{To cite this version:}

Cansen Jiang, Yohan Fougerolle, David Fofi, Cédric Demonceaux. Dynamic 3D Scene Reconstruction and Enhancement. IAPR 19th International Conference in Image Analysis and Processing (ICIAP17), Sep 2017, Catania, Italy. hal-01569314

\section{HAL Id: hal-01569314 https://hal.science/hal-01569314}

Submitted on 26 Jul 2017

HAL is a multi-disciplinary open access archive for the deposit and dissemination of scientific research documents, whether they are published or not. The documents may come from teaching and research institutions in France or abroad, or from public or private research centers.
L'archive ouverte pluridisciplinaire HAL, est destinée au dépôt et à la diffusion de documents scientifiques de niveau recherche, publiés ou non, émanant des établissements d'enseignement et de recherche français ou étrangers, des laboratoires publics ou privés. 


\title{
Dynamic 3D Scene Reconstruction and Enhancement
}

\author{
Cansen Jiang, Yohan Fougerolle, David Fofi, and Cédric Demonceaux \\ Le2i, FRE CNRS 2005, Arts et Métiers, Univ. Bourgogne Franche-Comté, France. \\ \{firstname.lastname\}@u-bourgogne.fr
}

\begin{abstract}
In this paper, we present a 3D reconstruction and enhancement approach for high quality dynamic city scene reconstructions. We first detect and segment the moving objects using 3D Motion Segmentation approach by exploiting the feature trajectories' behaviours. Getting the segmentations of both the dynamic scene parts and the static scene parts, we propose an efficient point cloud registration approach which takes the advantages of 3-point RANSAC and Iterative Closest Points algorithms to produce precise point cloud alignment. Furthermore, we proposed a point cloud smoothing and texture mapping framework to enhance the results of reconstructions for both the static and the dynamic scene parts. The proposed algorithms are evaluated using the real-world challenging KITTI dataset with very satisfactory results.
\end{abstract}

Keywords: 3D Reconstruction, 3D Scene Enhancement, Motion Segmentation, Point Cloud Registration

\section{Introduction}

For the past decades, 3D scene reconstruction has been widely studied due to the need of many applications, such as city map modelling [1], robot navigation [2], autonomous driving [3], etc. Among numerous works in this context, the most representative approaches are: structure-from-motion of image sequence [4], RGB-D data fusion [5], and laser scans registration [6]. These approaches make use of the common assumptions that the environments are mostly static or contain very few moving objects. However, such assumptions do not hold for many practical scenarios, such as crowed campus and markets.

To address the problem of $3 \mathrm{D}$ reconstruction of dynamic environments, in our previous works [7] [8], we proposed to detect and extract the moving objects prior to the scene reconstruction using a 2D-3D (RGB camera $+3 \mathrm{D}$ laser scanner) mobile camera system. Followed by, the static parts of the scene and the dynamic parts of the scene are independently reconstructed using a 3-point Random Sample Consensus (RANSAC) registration approach. Consequently, high quality static map and rigidly moving object reconstructions are achieved from highly dynamic environments. Since the 3-point RANSAC algorithm estimate the 3D-to-3D rigid transformation between two corresponding point sets, the accuracy of registration highly relies on the quality of corresponding sets. The 
3D-to-3D feature correspondences are established by the tracking of their associated 2D (image) features which is sensitive to noise, as detailed in Section IV [7]. Moreover, point cloud registration from long term observations inherently suffers from multi-layered problem due to the multiple scans of the same area. This problem can largely decrease the quality of the registration while increase the memory consumption. Building on top of [7] [9], in this work, we propose a more robust and effective algorithm, call Dual-Weighted Iterative Closest Point (DWICP) algorithm, and a 3D reconstruction enhancement framework is presented to produce photographic quality results of real outdoor scenes.

Point Cloud Registration: Iterative Closest Point (ICP) is one of the most commonly used algorithm due to its simplicity and robustness. However, the convergence of ICP algorithm requires a good initialization and rich geometric structures of the point clouds. For instance, ICP registration of two planar objects can easily fall into a local minimum. To overcome these problems, we exploit that an initialization using 3-point RANSAC registration algorithm is very effective. Moreover, a DW-ICP algorithm is introduced to iteratively estimate the rigid transformation by assigning different weights to the RANSAC inlier point pairs and the ICP correspondences, as detailed in Section 4.

3D Reconstruction Enhancement: Due to the measurement noise of data, the 3D registration from multiple observations has multi-layered artefacts. To address this problem, we employ a 3D Thin Plane Spline algorithm which smooths the object surface to a single layer. Furthermore, a ball pivoting surface triangulation approach is applied to construct 3D meshes of the smoothed point clouds. Finally, the textures of the 3D meshes are mapped and refined using mutual information, as detailed in Section 5.

\section{Related Work}

State-of-the-art methods in 3D point cloud registration are categorized as: ICPbased point cloud alignment [10] [11] [12], RANSAC-based [1] [7] [13] [14] point cloud registration, and volumetric representation-based point cloud fusion [5] [15] [16]. ICP-based methods are generally robust and accurate without prior knowledge of point-to-point correspondences. However, when the geometric structure of the point cloud is low, ICP registration yields to an ill-posed problem. RANSAC-based approaches are robust and efficient while they require sufficient number of precise 3D-to-3D matching pairs (at least $50 \%$ of them are inliers). Volumetric representation-based algorithms utilize the Signed Distance Function to describe the object surface using RGB-D camera. These methods work well for dense point cloud registration of large scene, but they suffer from over-smoothing problems.

\section{Dynamic Scene 3D Reconstruction}

In this section, we briefly revisit the principles of 3D reconstruction of dynamic scenes using 3D-based Sparse Subspace Clustering (3D-SSC) algorithm, 


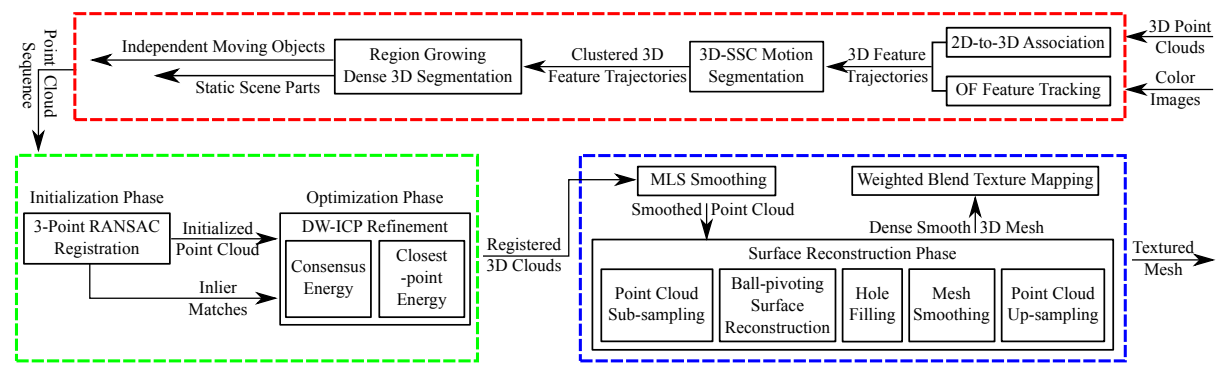

Fig. 1: Dynamic Scene 3D Reconstruction and Enhancement Framework: Red block segments the point cloud into the dynamic and the static scene parts. Green block registers the point cloud sequence using our DW-ICP algorithm. Blue block refines the registered point cloud, followed by the texture mapping.

see Fig. 1 Red Block. Given a mobile 2D-3D camera system, i.e. a car equipped with a 2D camera and a 3D laser scanner, our objective is to detect and extract the moving objects from a point cloud sequence, which yields to solve a Motion Segmentation (MS) problem. For this purpose, the 3D-SSC analyses the motion behaviours of the feature trajectories and segments them into independent motions. The principle of 3D-SSC is to construct an affinity matrix which encodes the similarity between the feature trajectories, followed by a spectral clustering algorithm to group the trajectories into their corresponding motion subspaces.

Let $\mathbf{X}=\left[\mathbf{x}_{1}, \cdots, \mathbf{x}_{F}\right]^{\top}$ be a vectorized 3D feature trajectory of $F$ frames, where $\mathbf{x}_{i}=[x, y, z] \in \mathbb{R}^{3}$ is a $3 \mathrm{D}$ feature point at frame $i$. Let $\mathbf{X}=\left[\mathrm{X}_{1}, \cdots, \mathrm{X}_{P}\right]$ be the assembly of $P$ feature trajectories belonging to $k$ different motions. Note that each independent motion determines a unique subspace. An element can be approximated by the linear combination of other elements from the same subspace, so called self-representation property. The self-representation model of MS problem is defined as a minimization problem:

$$
\min \|\mathbf{C}\|_{1,1} \quad \text { s.t. } \quad \mathbf{X}=\mathbf{X C}, \quad \operatorname{diag}(\mathbf{C})=0
$$

where $\mathbf{C}=\left[\mathrm{C}_{1}, \cdots, \mathrm{C}_{P}\right]$ is a square-sized sparse permutation matrix, and operator $\|\cdot\|_{1,1}$ denotes the $l_{1}-$ norm of each column of $\mathbf{C}$. The diagonal elements $\operatorname{diag}(\mathbf{C})$ of $\mathbf{C}$ are constrained as zeros to avoid the trivial solution, so that $\mathbf{X}_{i}$ cannot be used to represent $\mathrm{X}_{i}$ itself. More specifically, the sparse vector $\mathrm{C}_{i} \in \mathbb{R}^{P}$ contains a few of non-zero elements such that $\mathbf{X}_{i}=\mathbf{X} \mathbf{C}_{i}$. The sparsity of $\mathrm{C}_{i}$ constrains that the least number of closest feature trajectories are selected, which contributes to its robustness to noise and outliers. By minimizing Eq. (1), the desired sparse permutation matrix $\mathbf{C}^{*}$ is obtained. Afterwards, a symmetric affinity matrix $\mathbf{A}=\left|\mathbf{C}^{*}\right|+\left|\mathbf{C}^{*}\right|^{\top}$ is constructed to perform K-means spectral clustering to separate the $k$ independent motion subspaces. More details refer to [7]. 


\section{Robust Point Cloud Registration}

Point cloud registration from long term observations is a challenging problem. To tackle, we formulate an optimization problem that jointly minimizes both the feature matching energy and the nearest neighbour energy.

\subsection{3-Point RANSAC Registration}

Given a set of correspondences between two 3D point clouds, the rigid transformation parameters, i.e. $\mathrm{R}$ and $\mathrm{t}$, can be estimated by solving a linear system. Let $\mathbf{x}=[x, y, z]^{\top}$ and $\mathbf{y}=\left[x^{\prime}, y^{\prime}, z^{\prime}\right]^{\top}$ be two corresponding points under rigid transformation, denoted as $\mathbf{x}=\mathrm{Ry}+\mathrm{t}$. In which, $\mathrm{R}$ is a $3 \times 3$ rotation matrix and $\mathrm{t}$ is a $3 \times 1$ translation vector. Let $\mathrm{g}=\left[g_{x}, g_{y}, g_{z}\right]^{\top}$ be the Gibbs representation [17] of the rotation matrix $R$, we have $R=\left(I_{3}+G\right)^{-1}\left(I_{3}-G\right)$, where $G=[g]_{\times}$is the skew-symmetric matrix form of $\mathrm{g}$ and $\mathrm{I}_{3}$ is a $3 \times 3$ identity matrix.

By employing the Gibbs representation and the Cayley transform [18], the $3 \mathrm{D}$ registration problem is formulated as follow:

$$
\left[-[\mathbf{x}+\mathbf{y}]_{\times} \mathrm{I}_{3}\right]\left[\begin{array}{l}
\mathrm{g} \\
\tilde{\mathrm{t}}
\end{array}\right]=\mathbf{x}-\mathbf{y}
$$

where $[\cdot]_{\times}$denotes the skew-symmetric form of a vector and $\tilde{\mathrm{t}}=\left(\mathrm{I}_{3}+\mathrm{G}\right) \mathrm{t}$. Since each matching pair provides 2 independent equations, solving the 6 unknowns of Eq. (2) requires minimum 3 pairs of correspondences. For the sake of robustness to outliers, a RANSAC [19] framework is adopted, so called 3-Point RANSAC registration. The 3-point RANSAC point cloud registration algorithm is efficient and robust to outliers. However, in the presence of inaccurate correspondences, the quality of RANSAC registration is usually not very satisfactory. Therefore, we further propose to refine the registration by minimizing a dual-weighted closet-point energy taking into account both the RANSAC inlier matches as well as the full 3D point clouds.

\subsection{Robust ICP Registration}

When two overlapping point clouds of the same rigid object are given, the transformation between them is generally obtained by minimizing the energy derived from the closest-points distance. In most of the cases, this energy is minimized using an iterative method - also known as Iterative Closest Point (ICP) algorithm [20]. In each iteration, the ICP algorithm considers the closest points across two point clouds, say the reference and the model, as the corresponding ones. Let $\mathbf{X}=\left\{\mathbf{x}_{1}, \cdots, \mathbf{x}_{n}\right\}$ be the reference point cloud, and $\mathbf{Y}=\left\{\mathbf{y}_{1}, \cdots, \mathbf{y}_{m}\right\}$ be the new model, the robust method of ICP iteratively minimizes the following energy:

$$
\mathcal{E}_{I}(\hat{\mathbf{T}})=\min _{\mathbf{T}} \sum_{i=1}^{n} \rho\left(\min _{j \in\{1, \cdots, m\}}\left\|\mathbf{x}_{i}-\mathrm{T}_{\mathbf{j}}\right\|_{2}\right)
$$


where $\hat{T}$ is the desired transformation matrix that relates the two point clouds. Note that the energy term $\mathcal{E}_{I}$ includes a robust cost function to handle noisy and partial data. Our choice of robust cost, say $\rho(x)$, is the Tukey's biweight function [21]:

$$
\rho(x)=\left\{\begin{array}{cc}
\left(\tau^{2} / 6\right)\left(1-\left[1-(x / \tau)^{2}\right]^{3}\right) & \text { if }|x| \leq \tau \\
\left(\tau^{2} / 6\right) & \text { if }|x|>\tau
\end{array}\right.
$$

and the weight of each corresponding pair is defined by:

$$
w(x)=\frac{1}{x} \frac{\mathrm{d} \rho(x)}{\mathrm{d} x}=\left\{\begin{array}{cl}
{\left[1-(x / \tau)^{2}\right]^{2}} & \text { if }|x| \leq \tau \\
0 & \text { if }|x|>\tau
\end{array},\right.
$$

where $\tau$ is the inlier threshold, such that outliers $(|x|>\tau)$ are assigned with zero weights.

\subsection{Dual-Weighted ICP Registration}

While consensus-based registration method requires a subset of accurate correspondences, closest-point-based method requires rich structure of the point clouds. These prohibit us to make a choice of one method over another. Therefore, we propose to minimize a combined energy function - one from consensus, say $\mathcal{E}_{R}$, and the other from closest-point, say $\mathcal{E}_{I}$. We minimize the joint energy function in an iterative manner, named as dual-weighted ICP.

First, we define an energy function that measures the quality of the inlier set obtained from 3-point RANSAC. Note that due to the sparsity and noise, the inlier set obtained from RANSAC is not precise. Let $\left\{\mathbf{x}_{i} \leftrightarrow \mathbf{y}_{i}\right\}, i=1, \ldots, k$ be the inlier correspondence set, the energy $\mathcal{E}_{R}$ for matching consensus is expressed as:

$$
\mathcal{E}_{R}(\hat{\mathrm{T}})=\min _{\mathrm{T}} \sum_{i=1}^{k} \tilde{\rho}\left(\left\|\mathbf{x}_{i}-\mathrm{T} \mathbf{y}_{i}\right\|_{2}\right),
$$

where $k \leq m, n$, and $\tilde{\rho}(x)$ is the Huber's weight function denoted as:

$$
\begin{gathered}
\tilde{\rho}(x)=\left\{\begin{array}{cc}
\left(x^{2} / 2\right) & \text { if }|x| \leq \tilde{\tau} \\
\tilde{\tau}[|x|-(\tilde{\tau} / 2)] & \text { if }|x|>\tilde{\tau},
\end{array}\right. \\
\tilde{w}(x)=\frac{1}{x} \frac{\mathrm{d} \tilde{\rho}(x)}{\mathrm{d} x}=\left\{\begin{array}{cc}
1 & \text { if }|x| \leq \tilde{\tau} \\
(\tilde{\tau} /|x|) & \text { if }|x|>\tilde{\tau}
\end{array}\right.
\end{gathered}
$$

where $\tilde{\tau}$ is the threshold for inlier matches. The Huber loss function is selected under the assumption that the provided inlier set is noisy without severe outlier that needs to be completely discarded. In the spirit of Eq. (3) and Eq. (6), we formulate our combined energy function as follows: 


$$
\begin{array}{r}
\mathcal{E}(\hat{\mathbf{T}})=\min _{\hat{\mathbf{T}}}\left\{\alpha \sqrt{\frac{1}{n} \sum_{i=1}^{n} \rho\left(\min _{j \in\{1, \cdots, m\}}\left\|\mathbf{x}_{i}-\mathrm{T} \mathbf{y}_{j}\right\|_{2}\right)}+\right. \\
\left.(1-\alpha) \sqrt{\frac{1}{k} \sum_{i=1}^{k} \tilde{\rho}\left(\left\|\mathbf{y}_{i}-\mathrm{T} \mathbf{y}_{i}\right\|_{2}\right)}\right\},
\end{array}
$$

where $\alpha$ is the regularization term to control the influence of the $\mathcal{E}_{I}$ and $\mathcal{E}_{R}$ energy terms. Rather than optimizing the closest-point energy $\mathcal{E}_{I}$ or matching consensus energy $\mathcal{E}_{R}$ independently, the DW-ICP aims to iteratively and simultaneously optimize the joint energy $\mathcal{E}$ of Eq. (9).

\subsection{Discussions}

As summarized in Fig. 1 Green Block, our algorithm takes the 3-Point RANSAC registration as initialization. Afterwards, the DW-ICP is applied to refine the registration. Note that (also refer to Eq. (9)) the DW-ICP iteratively minimizes the combined energy of $\mathcal{E}_{R}$ and $\mathcal{E}_{I}$. On the one hand, $\mathcal{E}_{I}$ minimizes the overall registration error of the whole $3 \mathrm{D}$ point clouds. On the other hand, $\mathcal{E}_{R}$ minimizes the registration error of the inliers obtained form RANSAC. These two terms are usually complementary to each other, which is the key to the success of the proposed optimization framework. On top of the traditional ICP, there are two main advantages of our DW-ICP: (a) Feature matching constraint promises a proper registration regardless of the poor geometry structures of the point clouds. (b) Robust estimation framework is preserved such that the algorithm is generic and robust to outliers during a long term registration.

\section{3D Reconstruction Enhancement}

A complete pipeline for 3D reconstruction refinement is introduced to produce photo-realistic high quality 3D models, as shown in Fig. 1 Blue Block. There are three major steps involved, namely Moving Least Square (MLS) [22] point cloud smoothing, Surface Reconstruction [23], and Weighted Blend Texture Mapping [24]. Fig. 2 depicts the evolutions of a car object from raw registered point cloud to high quality textured mesh.

Point Cloud Smoothing: The registered point cloud from long term observation suffers from outliers and multi-layered effects due to the measurement noise and imperfect registrations. Surface reconstruction using such point cloud suffers from many visual artefacts, such as spiky surfaces and holes. Therefore, a MLS algorithm, which smooths an unorganized point could using a polynomial fitting, is applied due to its simplicity and effectiveness.

Surface Reconstruction: To avoid the redundant (overlapped) points caused by multiple observations, a sub-sampling processing is performed based on the 


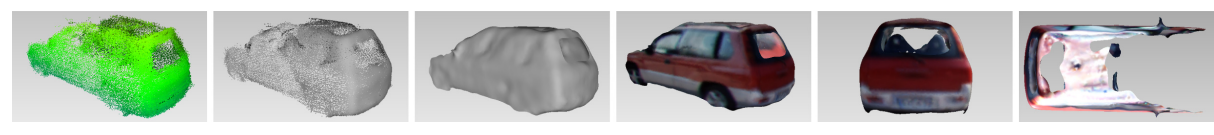

Fig. 2: Illustration of 3D Reconstruction Enhancement: from left to right are raw registration, smoothed point cloud, surface reconstruction, textured mesh in side view, back view and top view, respectively.

points' poisson-disk distribution [25]. Later on, a Ball Pivoting triangulation (or Poisson triangulation) algorithm is utilized to establish the neighbour-points relationships, followed by a dilation operation for hole closing. The Taubin Surface Smoothing [26] method is adopted to smooth the reconstructed surface while preserving the sharp edges. Finally, a Least Square Subdivision approach [27] is performed to refine and produce high quality meshes.

Texture Mapping: We make use of the 2D images for texture mapping. During this process, photographic alignment between the 3D mesh and the 2D images are required. Since the 2D-3D camera system is calibrated, and the motion of the camera is known, all the images are aligned with respect to the mesh reconstructed frame. The camera poses (between the cameras and the reconstructed mesh) are estimated by computing the inverse of the transformation matrices (obtained from registration) and using the camera calibration parameters. Furthermore, the blurring effect during the texture fusion from multiple images is reduced by using a Weighted Blending algorithm.

\section{Experiments}

We conducted experiments on both synthetic and real data (KITTI benchmark [28]). Since there is no ground truth data available for 3D reconstruction quantification, we generated three sets of synthetic data to quantify the robustness and accuracy of the proposed algorithms. Qualitative results of the proposed framework is presented using real data. All the experiments are conducted in a computer with Intel Quad Core i7-2640M, 2.80GHz, 8GB Memory. The algorithm parameters were set as: $\alpha=0.8, \tau=0.08 \mathrm{~m}, \tilde{\tau}=0.03 \mathrm{~m}$, rotation tolerance $\epsilon_{\mathrm{R}}=10 e-6$, translation tolerance $\epsilon_{\mathrm{T}}=10 e-6$, and max iteration as 100 .

Synthetic Datasets: The synthetic datasets were generated from three different objects, namely the Van, Red Car, and Cola Truck, see Fig. 4 for example. We simulate the motion behaviours the rigidly moving objects with smooth rotation and translation of 100 frames. Practical scenarios, such as partial overlaps, occlusions, and poor 3D geometric structures, are also taken into consideration. We applied 10 different levels of Gaussian noise, from 0.005 to 0.050 in meters. The maximum noise level is chosen as 2.5 times higher than the expected accuracy $(0.02 \mathrm{~m})$ of the Velodyne laser scanner. We compare the performances of the algorithms using the averaged absolute rotation and translation errors.

Fig. 5 shows the performances of 4 different algorithms, namely 3-Point RANSAC [7], RANSAC+ICP refinement [20], RANSAC+Robust-ICP [10] and 

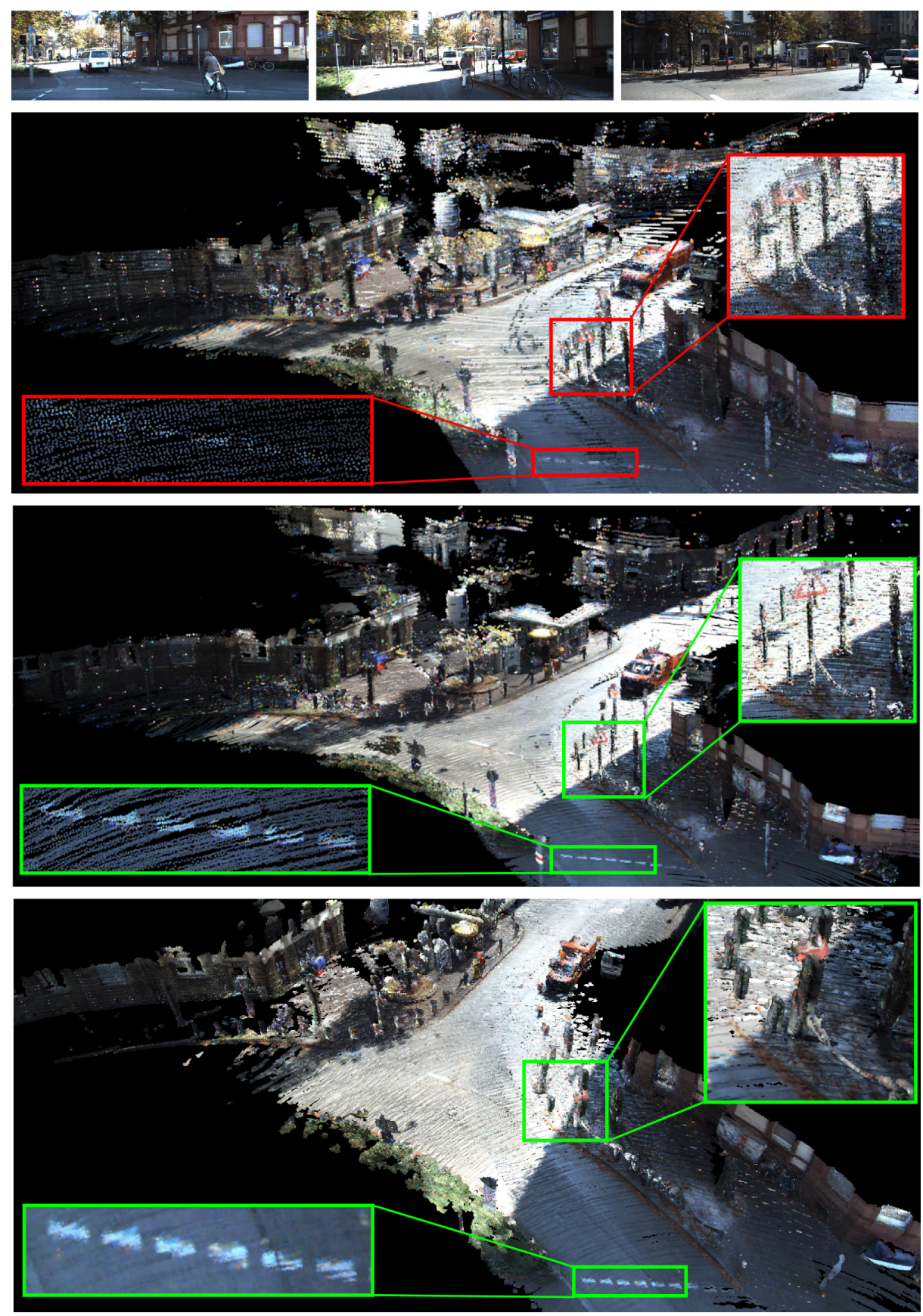

Fig. 3: High quality 3D reconstruction comparison: Row 1 are selected images. Row 2 is the 3D reconstruction using [7]. Row 3 is the $3 \mathrm{D}$ reconstruction of the proposed method, which is more accurate than [7]. Last row is the textured reconstructed 3D mesh of static scene parts, where details of small objects are lost as shown in the zoom-in region. 

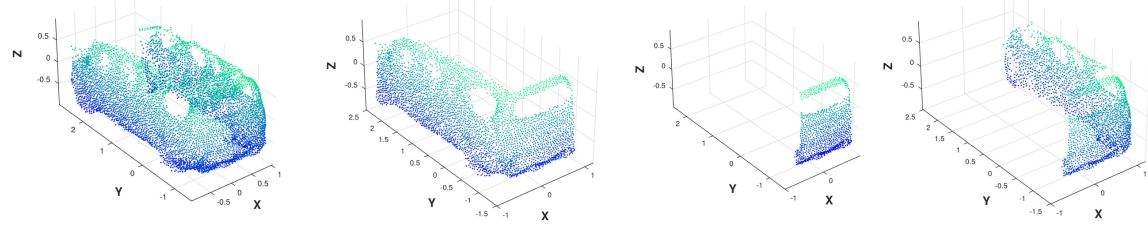

Fig. 4: Synthetic Van object with left, back and right side views.

RANSAC+DW-ICP. The overall performance of the algorithms are ranked (from top to down) as: DW-ICP, Robust-ICP, RANSAC+ICP and RANSAC. The Robust-ICP (using M-Estimator) has significantly better performance against that of traditional ICP. Most importantly, the proposed DW-ICP consistently outperforms the other approaches, regardless of rotation or translation.
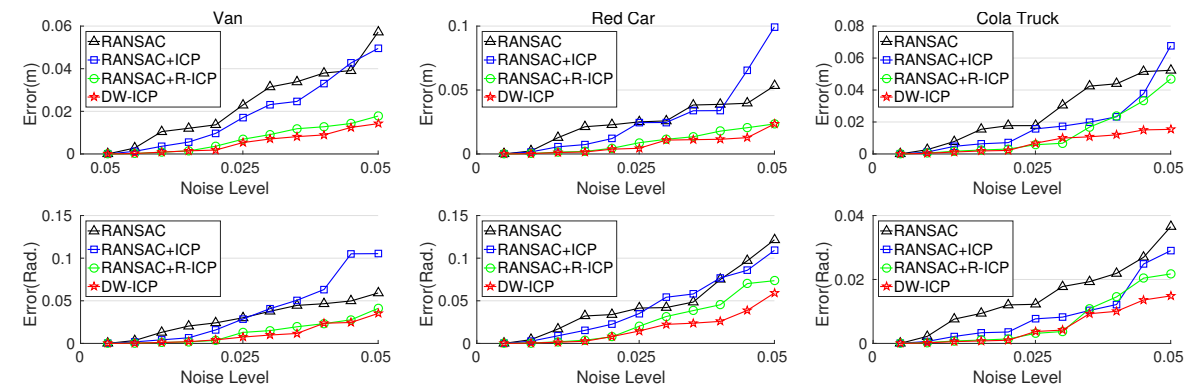

Fig. 5: Synthetic Data Quantification: top and bottom are averaged translation and rotation errors on Van, Red Car, and Cola Truck dataset, respectively.

Real Datasets: Table 1 depicts the dataset information, where the 3D Error (averaged Leave-One-Out Error) metric was used to quantify the registration performance. The registration error of our method is consistently lower than [7], although we have slightly more computation time. Moreover, the high quality reconstructions of Fig. 2 and Fig. 6 were obtained using the proposed framework of Fig. 1. Note that the objects are reconstructed from long-term and faraway observations (see Table 1). The framework effectively overcomes the accumulation errors during the registration process and products very satisfactory results. Moreover, Fig. 3 and 6 demonstrates that significant better registration quality of our method is achieved compared to [7].

\section{Conclusion and Future Work}

We have proposed an effective high quality 3D reconstruction and enhancement framework which is evaluated using both synaesthetic and realistic outdoor 
Table 1: Dataset Information: Col. Sides is number of object sides (left, right, back, and front) being captured. Col. Dist. is the averaged distance from the camera to the object. Col. 3-Point RANSAC [10] and Col. Ours show their respective averaged $3 \mathrm{D}$ error and computation time.

\begin{tabular}{|c|c|c|c|c|c|c|c|}
\hline \multirow{2}{*}{ Object } & \multirow{2}{*}{ \# Frame } & \multirow{2}{*}{ Sides } & \multirow{2}{*}{$\begin{array}{c}\text { Dist. } \\
(\mathrm{m})\end{array}$} & \multicolumn{2}{|c|}{ 3-Point RANSAC $[4]$} & \multicolumn{2}{|c|}{ Ours } \\
\cline { 5 - 8 } & & & Error $(\mathrm{m})$ & Time $(\mathrm{s})$ & Error $(\mathrm{m})$ & Time $(\mathrm{s})$ \\
\hline Van & 44 & 3 & 16.5 & 0.0150 & 3.1 & $\mathbf{0 . 0 1 3 1}$ & 4.6 \\
\hline Red Car & 60 & 3 & 10.8 & 0.0084 & 2.8 & $\mathbf{0 . 0 0 8 0}$ & 4.3 \\
\hline Cola Truck & 48 & 2 & 30.0 & 0.0234 & 3.7 & $\mathbf{0 . 0 2 2 9}$ & 4.1 \\
\hline
\end{tabular}

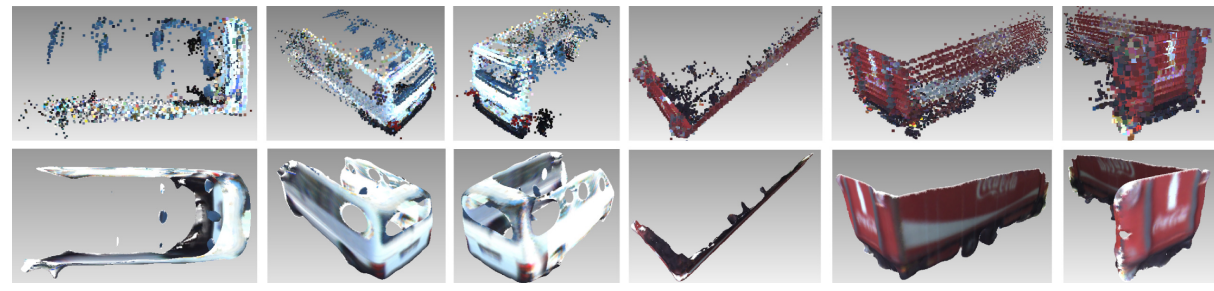

Fig. 6: Reconstructed Van and Cola Truck: top are registered point clouds using [7]; bottom are our high quality meshes.

dataset. The reconstructed 3D mesh of rigidly moving objects achieve photorealistic quality, while some small details of the large-scale 3D scene reconstruction are not well preserved. As future work, we expect to reconstruct the higher quality $3 \mathrm{D}$ mesh of the static scene parts.

\section{References}

1. Paudel, D.P., Demonceaux, C., Habed A., Vasseur, P. and Kweon, I.S.: 2D-3D camera fusion for visual odometry in outdoor environments. In IROS, 2014.

2. Castellanos, J.A. and Tardos, J.D.: Mobile robot localization and map building: A multisensor fusion approach. In Springer Science and Business Media, 2012.

3. Berger, C. and Rumpe, B.: Autonomous driving-5 years after the urban challenge: The anticipatory vehicle as a cyber-physical system. In ArXiv:1409.0413, 2014.

4. Pollefeys, M., Nistr, D., Frahm, J.M., Akbarzadeh, A., Mordohai, P., Clipp, B., Engels, C., Gallup, D., Kim, S.J., Merrell, P. and Salmi, C.: Detailed real-time urban 3d reconstruction from video. In IJCV, 78(2), pp.143-167, 2008.

5. Izadi, S., Kim, D., Hilliges, O., Molyneaux, D., Newcombe, R., Kohli, P., Shotton, J., Hodges, S., Freeman, D., Davison, A. and Fitzgibbon, A.: October. KinectFusion: real-time $3 \mathrm{D}$ reconstruction and interaction using a moving depth camera. In Proc. of ACM Symp. on User Interface Software and Technology, pp. 559-568, 2011.

6. Zhang, J. and Singh, S.: Low-drift and real-time lidar odometry and mapping. In Autonomous Robots, pp.1-16, 2016.

7. Jiang, C., Paudel, D.P., Fougerolle, Y., Fofi, D. and Demonceaux, C.: Static-Map and Dynamic Object Reconstruction in Outdoor Scenes Using 3-D Motion Segmentation. In IEEE Robotics and Automation Letters (RAL), 1(1), pp.324-331, 2016. 
8. Jiang, C., Paudel, D.P., Fougerolle, Y., Fofi, D. and Demonceaux, C.: Reconstruction 3D de scènes dynamiques par segmentation au sens du mouvemen, In Le 20èmé Congrès National sur la Reconnaissance des Formes et l'Intelligence Artificielle (RFIA), Clermont-Ferrand, France, June 2016.

9. Jiang, C., Christie, D., Paudel, D.P. and Demonceaux, C.: High Quality Recontruction of Dynamic Objects Using 2D-3D Camera Fusion. In Proc. of International Conference on Image Processing (ICIP), 2017.

10. Fitzgibbon, A.W.: Robust registration of $2 \mathrm{D}$ and $3 \mathrm{D}$ point sets. In Image and Vision Computing, 21(13), pp.1145-1153, 2003.

11. Pomerleau, F., Colas, F. and Siegwart, R.: A review of point cloud registration algorithms for mobile robotics. Foundations and Trends in Rob., pp.1-104, 2015.

12. Attia, M., Slama, Y. and Kamoun, M.A.: On Performance Evaluation of Registration Algorithms for 3D Point Clouds. In Proc. of Computer Graphics, Imaging and Visualization (CGiV), 2016.

13. Pankaj, D.S. and Nidamanuri, R.R.: A robust estimation technique for 3D point cloud registration. Image Analysis and Stereology, 35(1), pp.15-28, 2016.

14. Christie, D., Jiang, C., Paudel D.P., and Demonceaux C.: 3D reconstruction of dynamic vehicles using sparse 3D-laser-scanner and 2D image fusion. In Proc. of International Conference on Informatics and Computing (ICIC), 2016.

15. Yuheng Ren, C., Prisacariu, V., Murray, D. and Reid, I.: Star3d: Simultaneous tracking and reconstruction of 3d objects using rgb-d data. In ICCV, 2013.

16. May, S., Koch, P., Koch, R., Merkl, C., Pfitzner, C. and Nchter, A.: A Generalized 2D and 3D Multi-Sensor Data Integration Approach based on Signed Distance Functions for Multi-Modal Robotic Mapping. In VMV, pp. 95-102, 2014.

17. Gibbs, J.W.: Elements of vector analysis: arranged for the use of students in physics. Tuttle, Morehouse \& Taylor, 1884.

18. Diele, F., Lopez, L. and Peluso, R.: The Cayley transform in the numerical solution of unitary differential systems. Advances in Comp. Math., 8(4), pp.317-334, 1998.

19. Fischler, M.A. and Bolles, R.C.: Random sample consensus: a paradigm for model fitting with applications to image analysis and automated cartography. Communications of the ACM, 24(6), pp.381-395, 1981.

20. Besl, Paul J and McKay, Neil D.: Method for registration of 3-D shapes, RoboticsDL Tentative, pp. 586-606, 1992.

21. Cressie, N. and Hawkins, D.M.: Robust estimation of the variogram. In Jour. of the Intern. Asso. for Math. Geology, 12(2), pp. 115-125, 1980.

22. Lancaster, P. and Salkauskas, K.: Surfaces generated by moving least squares methods, Mathematics of Computation, 37(155), pp.141-158, 1981.

23. Bernardini, F., Mittleman, J., Rushmeier, H., Silva, C., Taubin, G.: The ballpivoting algorithm for surface reconstruction, IEEE Trans. on Visualization and Computer Graphics, 5(4), pp. 349-359, 1999.

24. Callieri, M., Cignoni, P., Corsini, M., Scopigno, R.: Masked photo blending: Mapping dense photographic data set on high-resolution sampled 3D models, Journal of Computers \& Graphics, 32(4) pp. 464-473, 2008.

25. Corsini, M., Cignoni, P., Scopigno, R., Efficient and flexible sampling with blue noise properties of triangular meshes, IEEE Trans. on Visualization and Computer Graphics, 18(6), pp.914-924, 2012.

26. Taubin, G.: Curve and surface smoothing without shrinkage. In ICCV, 1995.

27. Boyé, S., Guennebaud, G., Schlick, C.: Least squares subdivision surfaces, In Computer Graphics Forum, 29(7), pp.2021-2028, 2010.

28. Geiger, A., Lenz, P., Stiller, C. and Urtasun, R.: Vision meets robotics: The KITTI dataset. In IJRR, 32(11), pp.1231-1237, 2013. 